\title{
Effects of 5-azacytidine on RUNX3 gene expression and the biological behavior of esophageal carcinoma cells
}

\author{
SHUAI WANG ${ }^{1}$, HONG LIU $^{2}$, ZHOU WANG $^{1}$ and HUA-XIA CHEN ${ }^{1}$ \\ Departments of ${ }^{1}$ Thoracic Surgery and ${ }^{2}$ Otorhinolaryngology, Provincial Hospital Affiliated to Shandong University, \\ Jinan, Shandong 250021, P.R. China
}

Received June 7, 2013; Accepted January 28, 2014

DOI: $10.3892 / \mathrm{mmr} .2014 .1945$

\begin{abstract}
The present study investigated the effects of 5-azacytidine $(5$-azaC) on the expression level of the human runt-related transcription factor 3 (RUNX3) gene and the biological behavior of esophageal carcinoma Eca109 cells. The effect of the demethylation reagent 5 -azaC on the viability of Eca109 cells was detected by the MTT assay, which demonstrated that 5-azaC inhibited the viability of Eca109 cells in a time- and dose-dependent manner. Although demethylation of other genes may occur following treatment with 5-azaC, we focused on the RUNX3 gene. When treated with 5-azaC at hypoxic levels, the expression of RUNX3 increased and the methylation degree of the RUNX3 gene was decreased significantly in Eca109 cells. 5-azaC at $50 \mu \mathrm{M}$ demonstrated the highest RUNX3-induction activity, inducing RUNX3 mRNA and protein expression, and decreasing the degree of methylation of the RUNX3 gene. Methylation specific PCR indicated that 5-azaC induced RUNX3 expression through demethylation. The abilities of migration and invasion of Eca109 cells were inhibited by 5 -azaC. The growth of Eca109 cells treated with 5 -azaC in vivo was detected by a tumorigenesis experiment. 5-azaC inhibited the growth of Eca109 xenografts in nude mice. Taken together, our findings demonstrated that the RUNX3 gene is hypermethylated in Eca109 cells and that 5-azaC induces the expression of the RUNX3 gene by demethylation, which inhibits the proliferation, migration and invasion of Eca109 cells.
\end{abstract}

\section{Introduction}

For almost half a century, the incidence of esophageal cancer has continued to increase worldwide. East Asia (China, Japan and South Korea) has a much higher incidence of esophageal

Correspondence to: Professor Zhou Wang, Department of Thoracic Surgery, Provincial Hospital Affiliated to Shandong University, no. 324 Jingwu Road, Jinan, Shandong 250021, P.R. China

E-mail:wz620226@hotmail.com

Key words: 5-azacytidine, RUNX3 gene, demethylation, biological behavior, esophageal carcinoma cancer (1). Notably, the patient age at diagnosis is becoming younger (2). A variety of genetic alterations associated with esophageal cancer have been described, for example, p53, $\mathrm{Bcl}-2$, epidermal growth factor receptor, transforming growth factor- $\beta$ (3) and esophageal cancer-related gene 1 (4). The human runt-related transcription factor 3 gene (RUNX3) belongs to the runt domain family of transcription factors, which acts as a dominant tumor suppressor gene (TSG) in human solid tumors $(5,6)$. In 2002, RUNX3 was found to be a major growth regulator of human gastric cancer cells (7). Following this breakthrough, increasing evidence has demonstrated that a lack of RUNX3 expression is associated with the carcinogenesis and progression of diverse types of invasive cancer, including hepatocellular carcinoma (8), colorectal cancer (9) and breast cancer (10).

RUNX3, located on human chromosome 1p36.1, spans $\sim 67 \mathrm{~kb}$ and includes promoters 1-2, exons 1-6 and a $1290 \mathrm{bp}$ open reading frame that contains a highly conserved region designated as the 'runt domain' (11). A variety of abnormal changes in oncogenes and TSGs in the occurrence and development of human cancer have been identified. The expression of the RUNX3 gene can be downregulated by a loss of heterozygosity (7) or by mislocalization (12). However, large conserved cytosine phosphate guanine $(\mathrm{CpG})$ islands around promoters act as master regulators of gene expression (13). The RUNX3 gene is inactivated mainly by another mode, namely by the aberrant methylation of CpG islands. In Knudson's two-hit hypothesis, epigenetic silencing in the $\mathrm{CpG}$ island via hypermethylation is one of the mechanisms responsible for the dysfunction of genes. Previous studies have demonstrated that a lack of RUNX3 expression is associated with the positive $\mathrm{CpG}$ island methylator phenotype in gastric (13), breast (10) and hepatocellular cancer (13). In addition, the RUNX3 gene can be reactivated by a demethylation agent in the colorectal cell line, LoVo (14).

Smith et al (15) revealed that the RUNX3 gene was highly expressed in normal esophageal epithelial cells. However, expression of the RUNX3 gene in Barrett's esophagus tissues was significantly reduced. The loss of RUNX3 gene expression occurs in esophageal adenocarcinoma cells (15) and esophageal squamous carcinoma cells (16). However, the mechanism of inactivation of the RUNX3 gene in esophageal carcinoma remains controversial. The $\mathrm{CpG}$ islands around promoters suggest that RUNX3 is a target for epigenetic gene silencing 
in esophageal carcinogenesis and progression. However, there are limited data on the methylation status of RUNX3 in various types of human esophageal carcinoma cell lines. The present study investigated the expression of the RUNX3 gene induced by 5 -azacytidine (5-azaC) in Eca109 cells. The biological behavior of esophageal carcinoma cells pretreated with 5-azaC has not been studied. Therefore, we investigated the growth of esophageal squamous carcinoma cells in vivo by a tumorigenesis experiment in nude mice and further studied the mechanisms and effects of 5-azaC on Eca109 cells.

\section{Materials and methods}

Cell culture. The Eca109 human esophageal squamous carcinoma cells (obtained from the Institute of Cytobiology, Chinese Academy of Sciences, Shanghai, China) were maintained as monolayers in Roswell Park Memorial Institute medium (RPMI-1640; Gibco-BRL, Rockville, MD, USA) containing $10 \%$ heat-inactivated fetal calf serum, $100 \mathrm{U} / \mathrm{ml}$ of penicillin and $100 \mu \mathrm{g} / \mathrm{ml}$ of streptomycin at $37^{\circ} \mathrm{C}$ in a humidified incubator with $5 \% \mathrm{CO}_{2}$. Eca109 cells in the logarithmic growth phase were used for experiments. 5-azaC (Sigma, St. Louis, MO, USA) was dissolved in serum-free RPMI-1640 medium and stored at $-20^{\circ} \mathrm{C}$ until further use.

Cell viability assay. The viability of Eca109 cells was detected by an MTT assay (17). MTT (Sigma) was dissolved in PBS at $5 \mathrm{mg} / \mathrm{ml}$. Eca109 cells were seeded in 96-well microtiter plates (BD Biosciences, Franklin Lakes, NJ, USA) with $1 \times 10^{4}$ cells per well in $200 \mu$ l. Following being maintained in quiescence in a serum-free culture medium for $24 \mathrm{~h}, 5$-azaC was added at different final concentrations $(10-200 \mu \mathrm{M})$ in sextuplicate. MTT (50 $\mu \mathrm{l} ; 5 \mathrm{mmol} / \mathrm{l})$ was added at 6, 12, 24, 48 or $72 \mathrm{~h}$, respectively. Two control groups were included: one without 5-azaC treatment and one without cells. The optical density (OD) was measured at $490 \mathrm{~nm}$ in a microplate reader (SpectraMax M2; Molecular Devices, Sunnyvale, CA, USA). The inhibition rate (IR) of cell growth was calculated as: IR $=$ (the OD value of controls - the OD value of experimental groups) / the OD value of controls. The half maximal inhibitory concentration $\left(\mathrm{IC}_{50}\right)$ values were also calculated using Statistical Probit software (Probit Inc., Ottowa, ON, USA).

5-azaC intervention. Based on the results of the MTT analysis, Eca109 cells of three experimental groups (EG) were treated with 10,20 and $50 \mu \mathrm{M}$ of 5 -azaC for $72 \mathrm{~h}$, respectively, while Eca109 cells of the negative control (NC) group were treated without 5 -azaC for $72 \mathrm{~h}$, following $24 \mathrm{~h}$ of pre-culture.

Quantitative real-time RT-PCR ( $q R T-P C R)$. Total RNA was isolated from Eca109 cells using TRIzol reagent (Invitrogen Life Technologies, Carlsbad, CA, USA). According to the manufacturer's instructions, reverse transcriptase reactions were performed with $2 \mu \mathrm{g}$ of RNA, oligo-dT15 primer and M-MLV reverse transcriptase (Takara Bio, Inc., Shiga, Japan) in a volume of $20 \mu \mathrm{l}$. Gene-specific primers (Takara Shuzo Co., Kyoto, Japan) used were derived from PrimerBank and are summarized in Table I. The RUNX3 transcripts were analyzed by qRT-PCR, using the LightCycler 480 (Roche Diagnostics, Nutley, NJ, USA). The levels of the housekeeping gene $\beta$-actin were used as an internal control for the normalization of RNA quantity and quality differences in all samples. The real-time PCR reaction conditions were $30 \mathrm{sec}$ at $95^{\circ} \mathrm{C}$ followed by 45 cycles of $30 \mathrm{sec}$ at $94^{\circ} \mathrm{C}, 60 \mathrm{sec}$ at $30^{\circ} \mathrm{C}$ and $30 \mathrm{sec}$ at $72^{\circ} \mathrm{C}$. PCR reactions were terminated at $4^{\circ} \mathrm{C}$, following 7 min elongation at $72^{\circ} \mathrm{C}$. The measurements were performed in triplicates. The relative amount of mRNA was calculated as the calibrator normalized ratio using LightCycler 480 software 1.5 (Roche Diagnostics, Nutley, NJ, USA). The calibrator normalized ratio was measured using the following formula: $R Q=2^{-\Delta \Delta \mathrm{Ct}}$, where $\Delta \Delta \mathrm{Ct}=\left(\mathrm{Ct}_{\text {targeted gene }}-\mathrm{Ct}_{\beta \text {-actin }}\right)_{\text {targeted sample }}-\left(\mathrm{Ct}_{\text {targeted gene }}-\mathrm{Ct}_{\beta \text {-actin }}\right)$ calibration sample

Western blot analysis. The protein was extracted from cell lysate as described previously (18) and protein concentration was determined using the bicinchoninic acid (BCA) assay. Proteins were resolved on $10 \%$ SDS-PAGE and transferred onto nitrocellulose membranes. The membranes were inhibited for $1 \mathrm{~h}$ at room temperature with $5 \%$ non-fat dry milk containing $0.05 \%$ Tween-20 and $1 \%$ BSA, and incubated overnight at $4^{\circ} \mathrm{C}$ with primary antibodies against RUNX3 and against GAPDH (1:1,000 dilution; Santa Cruz Biotechnology, Inc., Santa Cruz, CA, USA). Following washing, the membranes were incubated with secondary antibody conjugated with horseradish peroxidase (HRP) anti-rabbit IgG (1:10,000; Santa Cruz Biotechnology, Inc.) for $1 \mathrm{~h}$ at room temperature. The bands were visualized by an enhanced chemiluminescence (ECL) detection system (LAS 4000 mini system; General Electric, Fairfield, CT, USA). The intensities of the acquired bands were measured by a computerized image analysis system (image reader LAS 4000; General Electric) and normalized to GAPDH as the endogenous control. RUNX3 expression was measured by the formula:

$$
\text { Relative Coefficient }=\frac{\text { Expression intensity of RUNX3 }}{\text { Expression intensity of GAPDH }}
$$

Methylation-specific PCR (MSP). Total genomic DNA was extracted from Eca109 cells. DNA was then subjected to sodium bisulfite modification and Wizard DNA Clean-up System purification, according to the manufacturer's instructions. Methylation-specific primers (Takara Shuzo Co.) are summarized in Table I. Briefly, the $20 \mu \mathrm{l}$ total reaction volume contained $(2 \mu \mathrm{l}) 100 \mathrm{ng}$ of DNA, 10X Ex Taq buffer $\left(\mathrm{Mg}^{2+}\right.$ Plus; $2 \mu \mathrm{l}), 2.5 \mathrm{mmol} / \mathrm{l}$ of dNTP mixture (1.6 $\mu \mathrm{l})$, Takara Ex Taq $(5 \mathrm{U} / \mu \mathrm{l}) 0.1 \mu \mathrm{l}$, sense and antisense primers (each at $1 \mu \mathrm{l}$; $100 \mathrm{ng})$. The reactions were hot-started at $97^{\circ} \mathrm{C}$ for $5 \mathrm{~min}$, prior to the addition of 0.75 units of Taq polymerase (Takara Shuzo Co.). The PCR conditions were as follows: 35 cycles of denaturation at $94^{\circ} \mathrm{C}$ for $20 \mathrm{sec}$, annealing at $60^{\circ} \mathrm{C}$ for $20 \mathrm{sec}$ and an extension at $72^{\circ} \mathrm{C}$ for $15 \mathrm{sec}$. PCR reactions were terminated at $4^{\circ} \mathrm{C}$, following $10 \mathrm{~min}$ elongation at $72^{\circ} \mathrm{C}$. Each set of PCR reaction products was loaded onto $2.5 \%$ agarose gels, stained with ethidium bromide and visualized under UV light

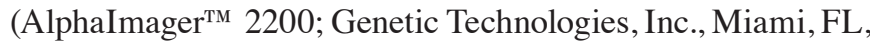
USA). The experiment was repeated three times.

Scrape motility assay. Eca109 cells pretreated in the presence or absence of 10,20 and $50 \mu \mathrm{M}$ of 5 -azaC for $72 \mathrm{~h}$ were plated at a density of $1 \times 10^{5}$ cells $/ \mathrm{ml}$ in 6 -well plates in RPMI-1640 
medium with $10 \%$ FBS. Following overnight incubation, the tip of a plastic pipette was drawn across the center of the well to produce a scraped area (19). The culture cells were washed twice with PBS, followed by incubation in RPMI-1640 medium. Immediately following scraping $(0 \mathrm{~h})$ and $72 \mathrm{~h}$ incubation, images of the two-dimensional Eca109 migration into the cell-free area at the center of the well were captured with an Olympus IX70 inverted microscope (Olympus Corporation, Tokyo, Japan) and printed. The cells that migrated into the scraped area following $72 \mathrm{~h}$ were counted by an observer blinded to the study design. The time it took for Eca109 cells to cover 6-well plates was noted. Each experiment was repeated four times and the average and standard error of the mean (SEM) were calculated.

Cell migration and invasion assay. As previously described (20), cell migration and invasion experiments were assayed in triplicate using a 24-well transwell setup (Merck KGaA, Darmstadt, Germany) and polycarbonate Nuclepore filters with an $8 \mu \mathrm{m}$ pore size. To assess the role of 5-azaC in migration and invasion, Eca109 cells were pretreated in the presence or absence of 10,20 and $50 \mu \mathrm{M}$ of 5 -azaC for $72 \mathrm{~h}$. Prior to each experiment, Eca109 cells were deprived of FBS during $24 \mathrm{~h}$. For invasion assays, the inserts were precoated with Matrigel (BD Biosciences, Erembodegem, Belgium) diluted 1:10 in serum-free Ham's F10 medium and the Matrigel was allowed to solidify for $1 \mathrm{~h}$ at $37^{\circ} \mathrm{C}$. For migration assays, the inserts were left uncoated. Each upper well was loaded with $2.5 \times 10^{5}$ cells in a total volume of $200 \mu \mathrm{l}$ of serum-free medium. The lower wells of the chamber were loaded with $600 \mu \mathrm{l}$ of RPMI-1640 medium with $10 \%$ FBS. The invasion assays were allowed to proceed for $24 \mathrm{~h}$ whereas migration assays were incubated for $6 \mathrm{~h}$. At the end of the experimental time period, any cells remaining on top of the insert were removed by scraping, while migrated cells attached to the underside were fixed for $10 \mathrm{~min}$ in methanol and stained with ethanol-based crystal violet solution. The cells were observed under a microscope (Leica DM4000 B; Leica, Wetzlar, Germany) in five random visual fields in the middle of the membrane.

Nude mouse xenograft model. A total of $50 \mathrm{BALB} / \mathrm{c}$ nude mice, including 25 females and 25 males, weighing 16-18 g and aged 4 weeks were purchased from Vital River Co. (certificate of quality no. SCXK; Beijing, China; 2012-0001) and maintained in specific pathogen-free facilities at the Experimental Animal Center of the Provincial Hospital Affiliated to Shandong University (Jinan, Shandong, China). The 50 nude mice were randomly divided into five groups: i) one black control (BC): the nude mice were injected with cell-free and serum-free culture medium; ii) one NC: the nude mice were injected with Eca109 cells of the negative group; iii) three EG: the nude mice were injected with Eca109 cells of three EG (as described in '5-azaC intervention'). The mice were provided with free access to food, water and bedding at all time points and were housed with a $12 \mathrm{~h}$ light/dark cycle in filter top cages containing a maximum of five mice per cage. The Eca109 cells were digested and suspended in serum-free culture medium. A total of $1 \times 10^{6}$ cells were injected into the skin of the right shoulder of 40 nude mice in the NC group and EG. Tumor volumes (V) were measured with an external caliper every 4 days and calculated by the formula: $V=\pi / 6 \mathrm{x}$ width $^{2}\left(\mathrm{~mm}^{2}\right) \mathrm{x}$ length $(\mathrm{mm})$ as described previously (21). The experiment was terminated in accordance with institutionally approved guidelines and tumors were harvested and weighed. The inhibition rate of tumor growth (IR) was calculated by the formula: IR = (tumor weight of $\mathrm{NC}$ - tumor weight of EG) / tumor weight of NC x $100 \%$.

Ethics statement. The present study was performed in strict accordance with the recommendations in the Guide for the Chinese Ethics Review Committees. The protocol was approved by the Ethics Committee of Shandong University (Jinan, Shandong, China).

Statistical analysis. The Statistical Package for the Social Sciences 13.0 (SPSS 13.0) was used for statistical analysis. Differences among different treatment groups were analyzed using the Student's t-test or one-way analysis of variance (ANOVA). Data are presented as the means $\pm \mathrm{SD}$. $\mathrm{P}<0.05$ was considered to indicate a statistically significant difference.

\section{Results}

Effects of 5-azaC on Ecal09 cell growth. Compared with the controls, 5-azaC inhibited Eca109 cell growth and the inhibition was dose and time dependent (Fig. 1). The $\mathrm{IC}_{50}$ of 5-azaC was $103.8 \mu \mathrm{M}$ at $72 \mathrm{~h}$, analyzed by Statistical Probit software. Thus, we selected 10, 20 and $50 \mu \mathrm{M}$ of 5 -azaC in further experiments to rule out the cytotoxicity of the drug itself.

5-azaC induces RUNX3 expression in Ecal09 cells. The mRNA levels of the RUNX3 gene in Eca109 cells of all three experiment groups were significantly higher than the negative control (Fig. 2A). There was an association between the expression of the RUNX3 gene and the concentration of 5-azaC. The 5-azaC at $50 \mu \mathrm{M}$ exhibited the greatest induction effect. Western blot analysis demonstrated the loss of RUNX3 protein expression in Eca109 cells and re-expression following treatment with 5 -azaC for $72 \mathrm{~h}$ at relatively low concentrations $(10-50 \mu \mathrm{M})$. The relative coefficient of expression of the RUNX3 protein was $0,0.196 \pm 0.004,0.278 \pm 0.013$ and $0.396 \pm 0.025$ in Eca109 cells pretreated with $0,10,20$ and $50 \mu \mathrm{M}$ of 5 -azaC for $72 \mathrm{~h}$, respectively. RUNX3 protein expression increased with sulforaphane concentration (Fig. 2B), which is consistent with the results of the RT-PCR.

RUNX3 is demethylated by 5-azaC in Ecal09 cells. The samples presenting methylated bands were scored as RUNX3 hypermethylation, whereas samples presenting unmethylated bands were considered to be positive for RUNX3 demethylation. RUNX3 hypermethylation was significantly higher in the negative group samples compared with the EG samples. Following treatment with 5-azaC, the RUNX3 gene was partially demethylated (Fig. 2C).

5-azaC inhibits the healing of a scrape wound. 5-azaC inhibited the motility of Eca109 cells in a dose-dependent manner, which was crucial in wound healing processes (Fig. 3A; Table II). The scrape wound healing time of Eca109 cells 
Table I. Primer sequences for RT-PCR and MSP.

\begin{tabular}{llll}
\hline Method & Gene & \multicolumn{1}{c}{ Upstream primer $\left(5^{\prime} \rightarrow 3^{\prime}\right)$} & \multicolumn{1}{c}{ Downstream primer $\left(5^{\prime} \rightarrow 3^{\prime}\right)$} \\
\hline RT-PCR & RUNX3 & ACCTGTCACAACGGCCAGAAC & TTCCAGTGAGGACAGGCCAGA \\
& $\beta$-actin & GGCGGCACCATGTACCC & AGGGGCCGGACTCGTCATACT \\
MSP & Methylated & AACGTTTTCGAGAAGGCGTAGCGC & CACGATACAAACCGAAACCATTCG \\
& RUNX3 & & \\
& Unmethylated & TGGGAATGTTTTTGAGAAGGTGTAGTGT & CACAATACAAACCAAAACCATTCA \\
& RUNX3 & & \\
\hline
\end{tabular}

RUNX3, runt-related transcription factor 3; MSP, methylation-specific PCR.

Table II. 5-azaC inhibits the migration and invasion of TE-1 cells.

\begin{tabular}{lccc}
\hline Assay & $0 \mu \mathrm{M}$ & $10 \mu \mathrm{M}$ & $20 \mu \mathrm{M}$ \\
\hline Scrape assay & & & $50 \mu \mathrm{M}$ \\
Migration (48 h) & $535 \pm 19.6$ & $318 \pm 15.6$ & $239 \pm 14.7$ \\
Migration (72 h) & $724 \pm 18.2$ & $387 \pm 17.8$ & $311 \pm 13.2$ \\
Transwell assay & & & $55 \pm 5.7$ \\
Migration (72 h) & $129 \pm 11.4$ & $79 \pm 8.1$ & $39 \pm 3.8$ \\
Invasion (72 h) & $99 \pm 8.9$ & $58 \pm 6.2$ & $14 \pm \pm 1.9$ \\
\hline
\end{tabular}

Number of migrating and invading TE-1 cells were calculated (mean \pm SEM). Differences between groups all have statistical significance $(\mathrm{P}<0.05)$. 5-azaC, 5-azacytidine.

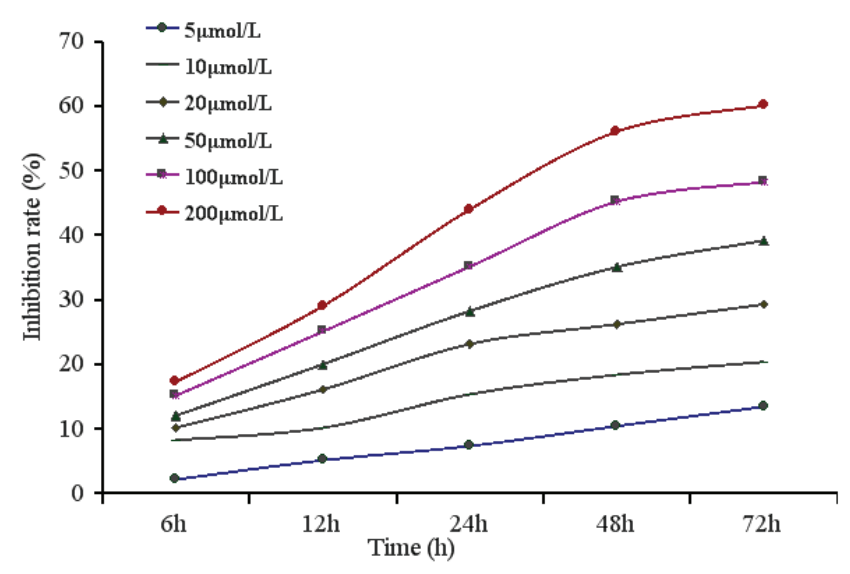

Figure 1. Eca109 cells were treated with increasing concentrations of 5-azaC. Cell viability was measured at various time points using the MTT assay. The results are expressed as the IR (\%) and are representative of three independent experiments. 5-azaC, 5-azacytidine; IR, inhibition rate.

pre-treated in the presence or absence of 10,20 and $50 \mu \mathrm{M}$ of 5-azaC for 72 h was 4, 5, 6 and 8 days, respectively.

5-azaC attenuates the migration and invasion of Ecal09 cells. Malignant Eca109 cells exhibited high migratory capability, by contrast, 5 -azaC attenuated the migratory capacity of Eca109 cells in a dose-dependent manner. 5-azaC at 10, 20 and $50 \mu \mathrm{M}$ decreased the migratory and invasive capability of Eca109 cells effectively and it had no detectable cytotoxicity (Fig. 3B; Table II).
5-azaC inhibits the growth of Ecal09 xenografts. There was no tumorigenesis in the $\mathrm{BC}$ group, while the tumorigenesis rate was $100 \%$ in the NC and EG (Fig. 4). The growth of tumor xenografts was stable in the first week following xenotransplantation. Tumor xenografts grew rapidly from the second week. The differences in tumor volume between the $\mathrm{NC}$ and EG was also increasingly significant. The mean tumor volumes of xenografts, 21 days later, were $3323 \pm 355 \mathrm{~mm}^{3}$ in the NC group and $1859 \pm 280,836 \pm 154$ and $412 \pm 94 \mathrm{~mm}^{3}$ in the 10,20 and $50 \mu \mathrm{M}$ of the 5-azaC EG, respectively (Fig. 4A). The weight of the xenografts was $2.62 \pm 0.41 \mathrm{~g}$ in the NC group and $1.95 \pm 0.22,1.22 \pm 0.16$ and $0.53 \pm 0.10 \mathrm{~g}(\mathrm{P}<0.05)$ in the 10 , 20 and $50 \mu \mathrm{M}$ of the 5 -azaC EG, respectively. The IR of tumor xenografts in the 10,20 and $50 \mu \mathrm{M}$ of the 5-azaC EG was 25.6, 53.4 and $79.8 \%$, respectively.

\section{Discussion}

Epigenetic change is an important mechanism of gene inactivation in all types of cancer, including DNA methylation, histone modification, chromatin remodeling, expression changes of non-coding RNA and genomic imprinting. DNA methylation is the most common and important mechanism of epigenetic change (22). The local hypermethylation of DNA resulting in the silencing of TSGs may be a mechanism for proliferation of aberrant cells in the early stages of human cancer (23). 5-azaC was developed as an anticancer drug (NSC-102876), which belongs to a class of pyrimidine ring analogs. In 5-azaC, the carbon 5 is replaced with nitrogen. When 5 -azaC binds DNA methyltransferase (DNMT) in an irreversible, covalent manner, 
A
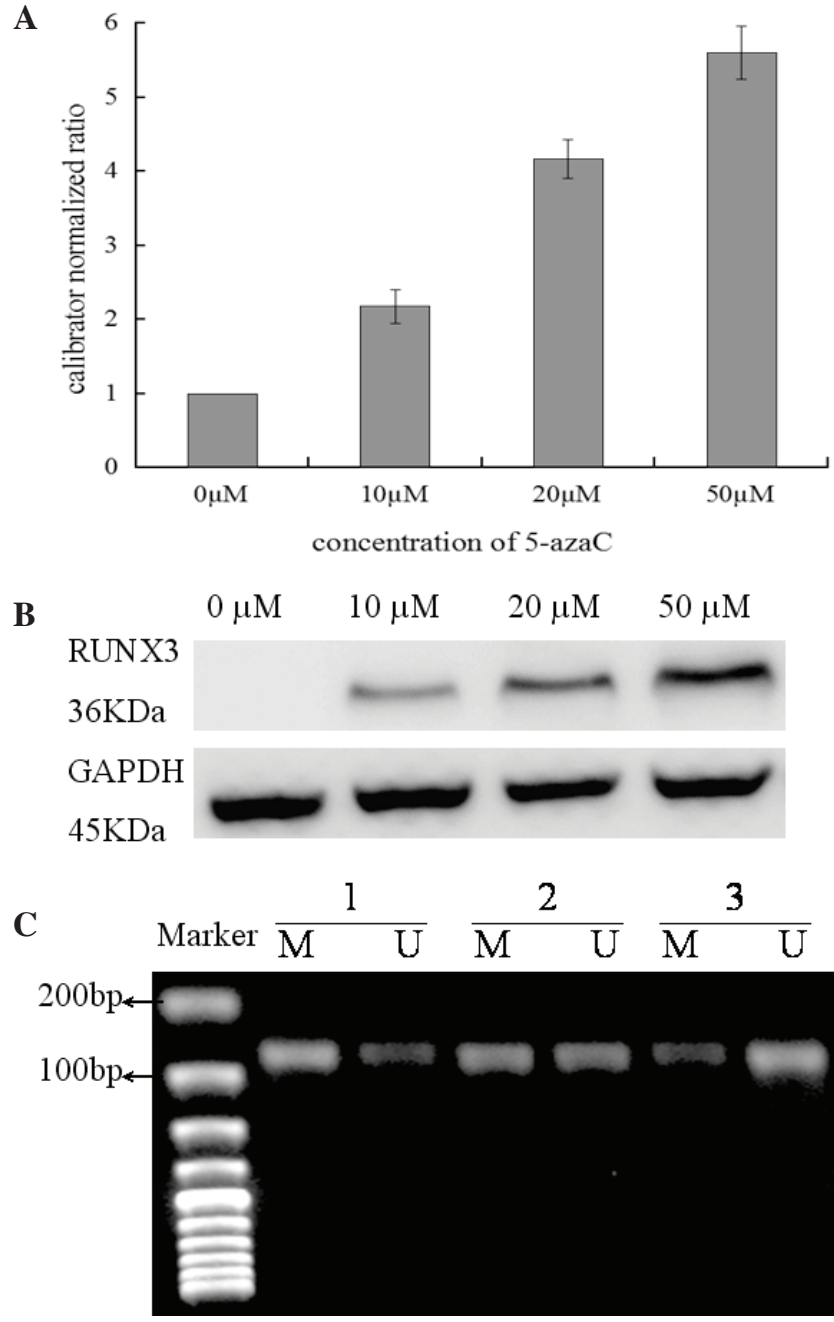

Figure 2. The expression of RUNX3 was induced by 5 -azaC by demethylation. Eca109 cells were pretreated without any drugs $(0 \mu \mathrm{M})$; Eca109 cells were pretreated with 10,20 and $50 \mu \mathrm{mol} / 1$ of 5 -azaC for $72 \mathrm{~h}(10,20$ and $50 \mu \mathrm{M}$ ). (A) Data are presented as the means \pm SD of the calibrator normalized ratio in triplicates. (B) The gel is representative of three independen experiments. (C) Lane 1, Eca109 cells were pretreated without any drugs; lane 2, Eca109 cells were pretreated with $20 \mu \mathrm{mol} / 1$ of 5 -azaC for $72 \mathrm{~h}$; lane 3, Eca109 cells were pretreated with $50 \mu \mathrm{mol} / 1$ of 5 -azaC for $72 \mathrm{~h}$. M, methylated sequence-specific PCR for RUNX3 (154 bp); U, unmethylated sequence-specific PCR for RUNX3 (158 bp); RUNX3, runt-related transcription factor 3; 5-azaC, 5-azacytidine.

it inhibits the methylation of genomic DNA and causes almost complete demethylation or hemi-demethylation. The cytotoxic and antineoplastic activities of 5-azaC have been demonstrated in leukemia (24) and other experimental tumors (25). The biological activities of 5-azaC include incorporating into DNA, depleting DNMT activities, preventing maintenance of the methylation state and altering chromatin structure (26). Consequently, 5-azaC induces re-expression of silenced, hypermethylated genes. In the present study, we investigated the effects of 5-azaC on the expression of the RUNX3 gene in Eca109 cells and the changes in cellular biological behaviors in vivo and in vitro.

In the present study, we first examined the effects of 5-AzaC on the proliferation of Eca109 cells using an MTT assay. The results demonstrated that 5 -azaC is cytotoxic to the Eca109 cells. Based on the $\mathrm{IC}_{50}, 103.8 \mu \mathrm{M}$ at $72 \mathrm{~h}$, we pretreated Eca109 cells at concentrations of 10,20 and $50 \mu \mathrm{M}$, to minimize the cytotoxicity of 5-azaC. We demonstrated that the RUNX3 gene was silenced in esophageal squamous carcinoma Eca109 cells. The same results were found in gastric (13), breast (10) and hepatocellular cancer (8). PCR and western blotting demonstrated that 5-azaC induced the expression of the RUNX3 gene. Furthermore, with higher concentrations, the expression of the RUNX3 gene was increased. Our experimental results are similar to a study by Long et al (16) in esophageal squamous carcinoma cells. However, in esophageal adenocarcinoma cells, the studies have different results (15). In esophageal adenocarcinoma OE33 cells, the expression of the RUNX3 gene can be induced by the DNMT inhibitor, 5 -azaC analog. In esophageal adenocarcinoma TE-7 cells, the expression levels of the RUNX3 gene were not significantly increased following 5-AzaC analog treatment. These different results appear to be due to the following reasons: RUNX3 gene expression may be inconsistent in esophageal carcinoma cell lines; the transcript levels of the gene are associated with environmental factors (e.g. the concentration of the DNMT inhibitor) and the detection method also has an effect on experimental results. This controversy reflects the diversity and complexity of RUNX3 gene expression in esophageal cancer cells.

Sugiura et al (27) detected the methylation status of the RUNX3 gene in esophageal adenocarcinoma TE1 to TE15 cell lines, however, hypermethylation was found only in the TE11 cell line (1/15). Among the 70 primary esophageal squamous cell carcinoma tissue samples, RUNX3 promoter methylation was detected in only four tissue samples (4/70). In the present study, the MSP assay indicated that the RUNX3 gene was hypermethylated and that 5-azaC was able to cause demethylation of the RUNX3 gene in Eca109 cells. Our findings are partially in disagreement with the study by Sugiura et al (27), however, they are similar to those of Long et al (16). In Eca109 cells, the loss of RUNX3 gene expression may result from hypermethylation. We also demonstrated that Eca109 cells can re-express the RUNX3 gene by demethylation.

Local and distant metastasis is one of the most important characteristics of a malignant tumor, particularly esophageal carcinoma. Metastasis is also the primary cause of clinical treatment failure. Tumor cell metastasis mainly involves the following processes: invasion of the surrounding tissue; access to the body circulation; distant spread; adhesion to endothelial cells or the matrix; deformation or movement; degradation of the extracellular matrix and engraftment or colonization. Therefore, the migratory and invasive abilities of tumor cells are particularly vital. To the best of our knowledge, this is the first study to evaluate the association between 5-azaC and the migratory and invasive abilities of Eca109 cells. Clearly, RUNX3 expression was higher in Eca109 cells pretreated with 5-azaC than the control. Scrape-wounding and transwell assays demonstrated that 5 -azaC impeded the movement, migration and invasion of Eca109 cells in a dose-dependent manner. The tumorigenesis assay of nude mice was designed to examine whether the intervention of 5-azaC altered the growth of Eca109 cells in vivo. Although the growth of xenografts was not completely suppressed by 5 -AzaC, the tumor volume and weight of the EG group were significantly lower than those of the NG group $(\mathrm{P}<0.05)$. The IR of 5 -azaC was $79.8 \%$ at a concentration of $50 \mu \mathrm{M}$. 
A

$\mathrm{Oh}$

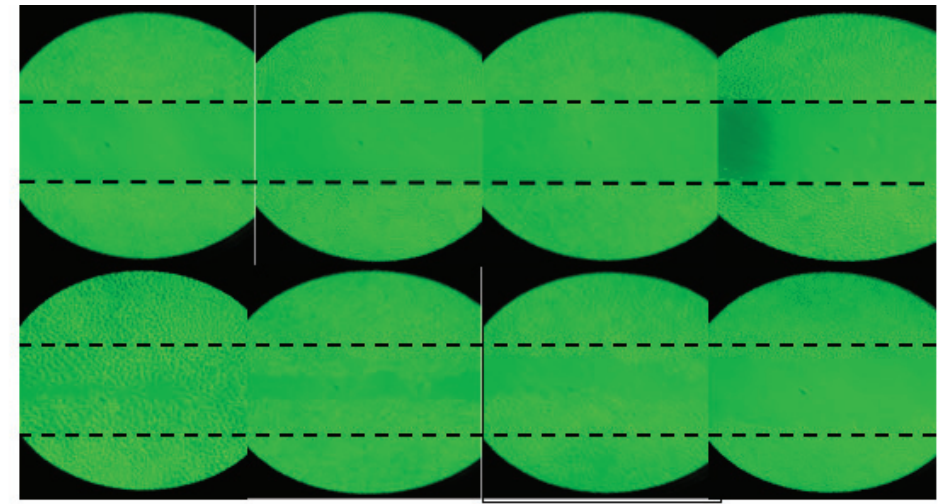

$0 \mu \mathrm{M}$

$10 \mu \mathrm{M}$

$20 \mu \mathrm{M}$

$50 \mu \mathrm{M}$

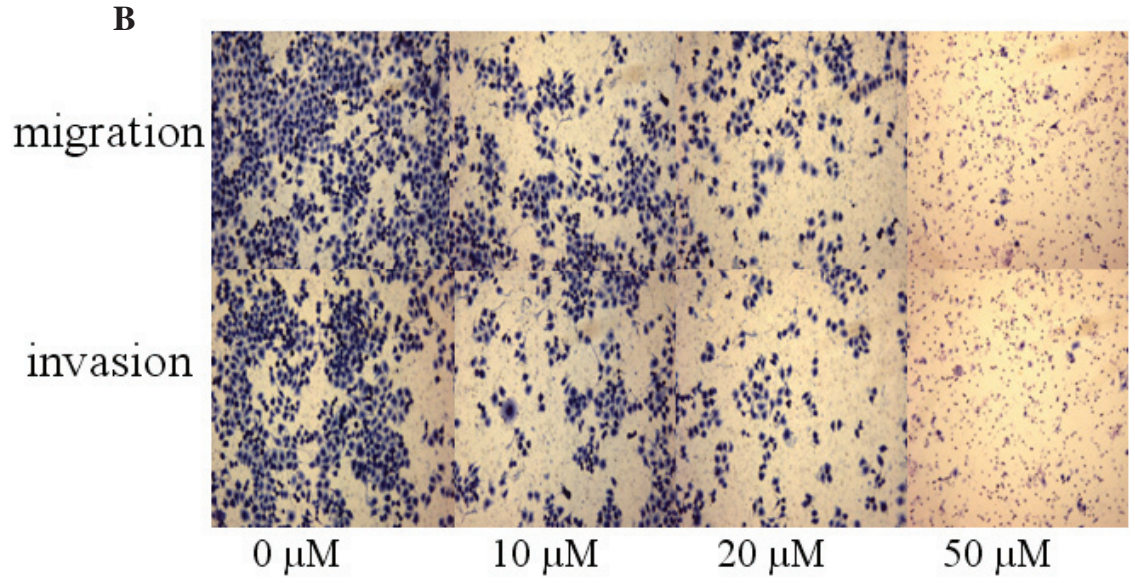

Figure 3. Migration and invasion of Eca109 cells was inhibited. Eca109 cells were pretreated with $0,10,20$ and $50 \mu \mathrm{mol} / 1$ of 5 -azaC for $72 \mathrm{~h}(0,10,20$ and $50 \mu \mathrm{M}$ ). (A) Representative images of four separate scrape wound assays at 0 and $72 \mathrm{~h}$ (magnification, $\mathrm{x} 4$ ). (B) Representative images of the migrating and invading cells in five separate transwell assays (magnification, x10). 5-azaC, 5-azacytidine.
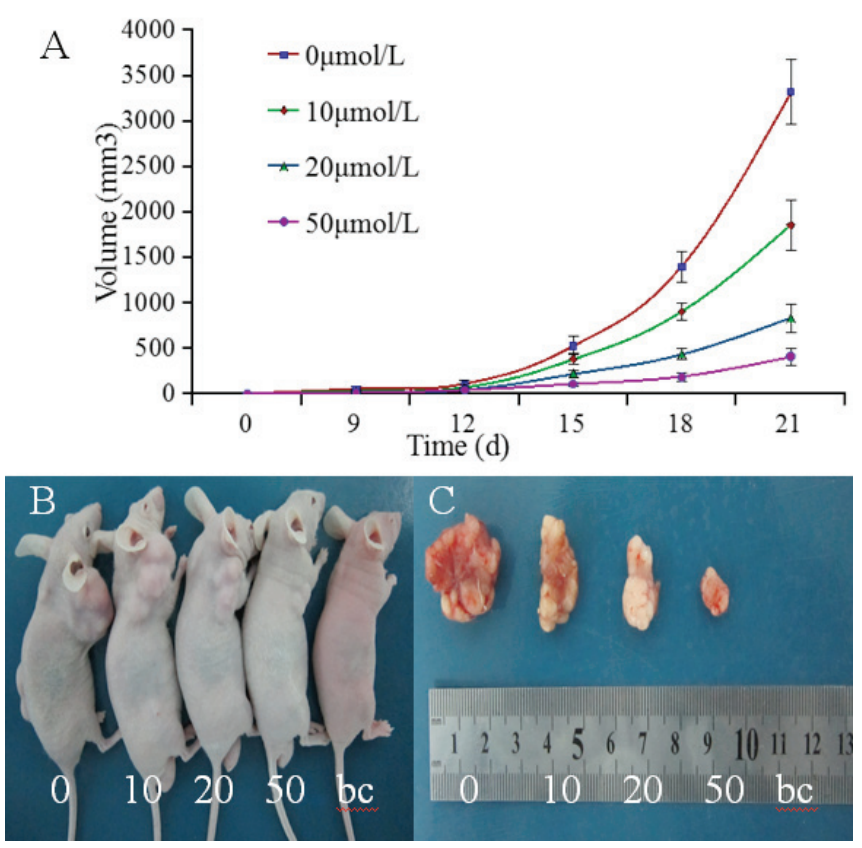

Figure 4. Tumorigenicity of Eca109 cells in nude mice was significantly inhibited. (A) Tumor volumes are presented as a growth curve. (B) Representative images of the nude mice at 21 days following injection. (C) Representative images of the tumors excised from mice. Nude mice $(0,10,20$ and 50) were injected with Eca109 cells pretreated with 0,10,20 and $50 \mu \mathrm{mol} / 1$ of 5-azaC for $72 \mathrm{~h}$, respectively; bc (black control). The nude mice were injected with PBS. 5-azaC, 5-azacytidine.
The RUNX3 gene was able to be activated by 5 -azaC via demethylation in Eca109 cells. However, 5 -azaC is a nonspecific DNMT inhibitor. Following treatment with 5-azaC, the promoter methylation status of other genes in Eca109 cells may be changed simultaneously. We focused on the RUNX3 gene in the present study. The RUNX3 gene was involved in the effects of 5-azaC on migration, invasion and the growth of esophageal carcinoma in vitro and in vivo. The regulation of RUNX3 expression and the malignancy of Eca109 cells is intricate. We demonstrated that reversible hypermethylation was one of the potential mechanisms of RUNX3 deficiency in Eca109 cells. We also demonstrated that the upregulation of the RUNX3 gene was one of the mechanisms by which 5-azaC inhibited the proliferation, migration and invasion of Eca109 cells.

The present study supports previous studies which state that the aberrant hypermethylation of TSGs is a common event that occurs in esophageal carcinoma and suggests that certain TSGs may be specific markers for the diagnosis and prognosis of esophageal carcinoma. In esophageal carcinoma, the intervention of RUNX3 methylation as a tumor treatment strategy has wide prospects for clinical application.

\section{Acknowledgements}

This study was supported by a grant from the Natural Science Foundation of China (no. 81172161). 


\section{References}

1. Kamangar F, Dores GM and Anderson WF: Patterns of cancer incidence, mortality, and prevalence across five continents: defining priorities to reduce cancer disparities in different geographic regions of the world. J Clin Oncol 24: 2137-2150, 2006.

2. Trivers KF, Sabatino SA and Stewart SL: Trends in esophageal cancer incidence by histology, United States, 1998-2003. Int J Cancer 123: 1422-1428, 2008.

3. Shibata-Kobayashi S, Yamashita H, Okuma K, Shiraishi K, Igaki H, Ohtomo K and Nakagawa K: Correlation among 16 biological factors [p53, p21(waf1), MIB-1 (Ki-67), p16(INK4A), cyclin D1, E-cadherin, Bcl-2, TNF- $\alpha$, NF- $\kappa$ B, TGF- $\beta$, MMP-7, COX-2, EGFR, HER2/neu, ER, and HIF-1 $\alpha$ ] and clinical outcomes following curative chemoradiation therapy in 10 patients with esophageal squamous cell carcinoma. Oncol Lett 5: 903-910, 2013.

4. Rasool S, Khan T, Qazi F and Ganai BA: ECRG1 and its relationship with esophageal cancer: a brief review. Onkologie 36: 213-216, 2013.

5. Zhang YW, Eom SY, Yim DH, et al: Evaluation of the relationship between dietary factors, CagA-positive Helicobacter pylori infection, and RUNX3 promoter hypermethylation in gastric cancer tissue. World J Gastroenterol 19: 1778-1787, 2013.

6. Li M, Tan SY, Zhang J and You HX: Effects of paeonol on intracellular calcium concentration and expression of RUNX3 in LoVo human colon cancer cells. Mol Med Rep 7: 1425-1430, 2013.

7. Li QL, Ito K, Sakakura C, et al: Causal relationship between the loss of RUNX3 expression and gastric cancer. Cell 109: 113-124, 2002.

8. Park WS, Cho YG, Kim CJ, et al: Hypermethylation of the RUNX3 gene in hepatocellular carcinoma. Exp Mol Med 37: 276-281, 2005.

9. Ku JL, Kang SB, Shin YK, et al: Promoter hypermethylation downregulates RUNX3 gene expression in colorectal cancer cell lines. Oncogene 23: 6736-6742, 2004.

10. Subramaniam MM, Chan JY, Soong R, et al: RUNX3 inactivation by frequent promoter hypermethylation and protein mislocalization constitute an early event in breast cancer progression. Breast Cancer Res Treat 113: 113-121, 2009.

11. Bangsow C, Rubins N, Glusman G, et al: The RUNX3 gene--sequence, structure and regulated expression. Gene 279: 221-232, 2001 .

12. Ito K, Liu Q, Salto-Tellez M, et al: RUNX3, a novel tumor suppressor, is frequently inactivated in gastric cancer by protein mislocalization. Cancer Res 65: 7743-7750, 2005.

13. Tang GH, Sun SW and He XS: Correlation of CpG methylation status of Runx3 with pathogenesis of gastric carcinoma. Zhonghua Bing Li Xue Za Zhi 41: 314-319, 2012 (In Chinese).
14. Deng $\mathrm{T}$ and Zhang $\mathrm{Y}$ : Possible involvement of activation of P53/P21 and demethylation of RUNX 3 in the cytotoxicity against Lovo cells induced by 5-Aza-2'-deoxycytidine. Life Sci 84: 311-320, 2009.

15. Smith E, De Young NJ, Pavey SJ, et al: Similarity of aberrant DNA methylation in Barrett's esophagus and esophageal adenocarcinoma. Mol Cancer 7: 75, 2008.

16. Long C, Yin B, Lu Q, et al: Promoter hypermethylation of the RUNX3 gene in esophageal squamous cell carcinoma. Cancer Invest 25: 685-690, 2007.

17. Alvarez-Díaz S, Larriba MJ, López-Otín C and Muñoz A: Vitamin D: Proteases, protease inhibitors and cancer. Cell Cycle 9: 32-37, 2010

18. Wang M, Chen S, Wang S, et al: Effects of Phytochemicals sulforaphane on uridine diphosphate-glucuronosyltransferase expression as well as cell-cycle arrest and apoptosis in human colon cancer Caco-2 cells. Chin J Physiol 55: 134-144, 2012.

19. Zhao C, Fernandes MJ, Prestwich GD, et al: Regulation of lysophosphatidic acid receptor expression and function in human synoviocytes: implications for rheumatoid arthritis? Mol Pharmacol 73: 587-600, 2008.

20. Shi GZ, Yuan Y, Jiang GJ, et al: PRAF3 induces apoptosis and inhibits migration and invasion in human esophageal squamous cell carcinoma. BMC Cancer 12: 97, 2012.

21. Perera Y, Farina HG, Hernández I, et al: Systemic administration of a peptide that impairs the protein kinase (CK2) phosphorylation reduces solid tumor growth in mice. Int J Cancer 122: 57-62, 2008.

22. Grønbaek K, Hother C and Jones PA: Epigenetic changes in cancer. APMIS 115: 1039-1059, 2007.

23. Park SY, Kim BH, Kim JH, et al: Methylation profiles of CpG island loci in major types of human cancers. J Korean Med Sci 22: 311-317, 2007.

24. Aimiuwu J, Wang $\mathrm{H}$, Chen $\mathrm{P}$, et al: RNA-dependent inhibition of ribonucleotide reductase is a major pathway for 5-azacytidine activity in acute myeloid leukemia. Blood 119: 5229-5238, 2012.

25. Acun T, Oztas E, Yagci T and Yakicier MC: SIP1 is downregulated in hepatocellular carcinoma by promoter hypermethylation. BMC Cancer 11: 223, 2011.

26. Kiziltepe T, Hideshima T, Catley L, et al: 5-Azacytidine, a DNA methyltransferase inhibitor, induces ATR-mediated DNA double-strand break responses, apoptosis, and synergistic cytotoxicity with doxorubicin and bortezomib against multiple myeloma cells. Mol Cancer Ther 6: 1718-1727, 2007.

27. Sugiura H, Ishiguro H, Kuwabara Y, et al: Decreased expression of RUNX3 is correlated with tumor progression and poor prognosis in patients with esophageal squamous cell carcinoma. Oncol Rep 19: 713-719, 2008. 